\title{
On The Occurrence of Historical Pandemics During The Grand Solar Minima
}

\author{
Carlos E. Navia
}

\section{ABSTRACT}

\begin{abstract}
The occurrence of viral pandemics depends on several factors, including their stochasticity, and the prediction may not be possible. However, we show that the historical register of pandemics coincides with the epoch of the last seven grand solar minima of the Holocene era. We also included those more recent, and some pandemics incidence forecasts for the coming years, with the probable advent of a new Dalton-like solar minimum with onset in 2006. Taking into account that cosmic-rays and consequently the neutrons produced by them in the atmosphere are in an inverse relationship with the solar activity. We show the possibility of abstention the pandemics occurrence rates considering that they are due to mutations induced by the neutron capture upon the presence of hydrogen in the viral proteins, producing radical changes, an "antigenic-shift", forming a new type of viral strains. Since the cross-section of neutron capture is small, the occurrence of an antigenic-shift requires a substantial increase in the flow of thermal neutrons, and this is more feasible during the epochs of the grand solar minima when the galactic cosmic-rays fluence is highest. On the other hand, the rate of occurrence of the most common viral outbreaks (epidemics) suggests a link with the scattering of neutrons and other secondary cosmic rays, causing small changes, an "antigenic-drift". In addition to being influenced by seasonal conditions, we also show that antigenic-drift variations are modulated by the 11-year solar cycle. We present details of these observations.
\end{abstract}

Keywords: neutron-induced mutations, cosmic rays modulation, sun-activity.

Published Online: July 29, 2020

ISSN: 2684-4451

DOI : $10.24018 /$ ejphysics.2020.2.4.11

Carlos E. Navia*

Instituto de Física, Universidade Federal

Fluminense, Brazil.

(e-mail: navia@if.uff.br)

\section{INTRODUCTION}

From the '70s, a series of scientific articles [1], [2], suggest correlations of viral outbreaks and sunspots. In all cases, the connection is between the influenza outbreaks and the solar cycle maxima and indicates that the hight solar activity is their origin. However, this connection is controversial because many viral outbreaks occurred during solar minimums. Data from 1728 to 1936, collecting the dates from 29 influenza outbreaks [3] show that only about 58\% of outbreaks occur around the solar maxima. Even so, using more sophisticated statistical methods to obtain a more accurate relationship between influenza outbreaks and the peak of the solar cycles, showing no statistically significant evidence of any association [4].

In general, viruses are constantly changing, by random genetic mutations (spontaneous mutations) or probably due to mutations induced by an external agent. The most common are those producing small changes, are known as "antigenicdrift" [5], which leads to changes in proteins, but only on the surface of the virus. Antigenic drift can happen continuously (yearround) as the virus replicates, but with seasonal variations impacting host-pathogen interactions [6].

However, the virus can have a radical change, called "antigenic-shift" [7], probably induced by an external agent; the change can result in a new protein, a new virus subtype. An outbreak of this type of infection depending on its aggressiveness and spread can trigger pandemics, because the human body does not yet have immunity to a new strain of the virus.

On the other hand, the production of mutations by "artificial" neutron in the male Drosophila melanogaster [8] showed that fast neutrons $(0.6 \mathrm{MeV})$ produce mutation rates about three times higher than the mutation rates by other types of radiation. And the latter does not differ from mutation rates, without any irradiation ("spontaneous" mutations).

A spontaneous mutation occurs naturally (apparently) without any external agent and may be due to errors in DNA replication, genetic defects in cells, and so on. However, spontaneous mutations occur at low rates, and sometimes they cannot explain alone the frequencies of mutations that occur in some biological systems, without being exposed to any external agents.

A viable alternative is to consider that the neutrons produced in the atmosphere from cosmic rays interaction are the primary source of the background levels of neutron radiation at ground level, inducing viral mutations. Neutrons at ground level are in a wide range of energies from $0.1 \mathrm{eV}$ (thermal neutrons) to $10^{8} \mathrm{eV}$, with an almost flat energy spectrum, but with enhancement by a factor of six, around 1 
- $10 \mathrm{MeV}$ (fast neutrons) [9]. Thus, neutrons from cosmic rays can explain from damages in computer memories [10], to inducing mutations in biological systems.

In this paper, we show the possibility to obtain that the viral outbreaks and pandemics occurrence rates, considering that they are due to mutations induced by the cosmic neutrons, upon the presence of hydrogen in the viral proteins. We link viral outbreaks to neutron and other secondary cosmic rays scattering, producing small changes, an "antigenic-drift". So, pandemics would be induced by mutations caused by the neutron capture's, the neutron capture produces an ion trail in the path of the recoil protons. The effect would be a radical change, an "antigenic-shift", forming a new type of viral strains.

In-situ observations of cosmogenic nuclide concentration in organic remains and in glaciers are strong evidence on the flow of cosmic rays on Earth in past times, and from it, obtain information on the long-term variations of solar activity. Cosmogenic nuclide observation, allow obtaining information, for example, of the occurrence of five grand minima of solar activity in the last millennia. These grand minima are favorable epochs to the incidence of pandemics because the increase of the cosmic-neutrons intensity for long periods increases the probability of antigenic-shift in already existent viruses. It can explain why pandemics are occurring preferentially in these periods.

Most scientific literature indicates that periods of high solar activity are bad for the health of living beings. There is experimental evidence; for example, excessive UV radiation is related to skin cancer, the correlation between the variation of sunspots and the number of whales stranded on the beaches [11]. Also, the solar activity would be responsible for an increase in the cases of catastrophic events, such as suicides, homicides, cardiac diseases and traffic accidents, and so on [12], [13]. However, here we postulate and will try to show the opposite, those periods of lower solar activity, sometimes lasting for more two solar cycles, maybe could potentially be more dangerous for human health.

We show that in periods of lower solar activity, there is an increase in the global cosmic-ray neutron dose rates, causing an increase in the rate of antigenic-drifts and especially the antigenic-shift of already existent viruses. It can also explain why the periods of lower solar activity are promising for the appearance of viral pandemics. The new viral antigenicshift can find the human body as a host, increasing their reproductive capacity and survival rates. Below, we detail these observations.

\section{COSMIC-NEUTRONS ON EARTH}

\section{A. Neutrons from galactic cosmic rays}

According to Solar Dynamo theory [14], the 11-year sunspot cycle is due to an oscillatory exchange of energy between toroidal and poloidal magnetic fields and stochastic variations between consecutive cycles. However, if the changes between the solar cycles are stochastic, it is still an open question [15]. They probably follow a certain regularity with a periodicity higher than 11 years. Indeed, studies are indicating, for example, the 88-year Gleissberg solar cycle [16], the 207-year Sues solar cycle [17], and the 1000-year
Eddy solar cycle [18] and that could explain at least partially the existence of periods of high and low solar activity, with duration larger than 11 years.

On a large scale, the propagation of galactic cosmic rays (GCRs) is almost isotropic. While inside of the heliosphere, the deflection of the GCRs by the solar magnetic field makes their spread not isotropic. As the energy of the GCR decreases, the deflection is more accentuates; that is, the GCRs are subject to solar modulation.

In the vicinity of the Earth, the GCRs present several anisotropies in their propagation; there are parallel and perpendicular gradients to the ecliptic plane. They are responsible for the solar diurnal anisotropy, among others, known as shortterm modulations [19]. However, there is also the long-term modulation, and the main is the 11-years sunspot solar cycle, responsible for the main intensity variation of the GCRs at Earth.

Earth's geomagnetic field sharply deflects low-momentum per unit charge (magnetic rigidity) cosmic rays particles. The deflection increases as the rigidity decreases and only particles above a specific geomagnetic rigidity cutoff can reach the upper atmosphere. The geomagnetic cutoff depends on the geomagnetic latitude. The magnetic cutoff effect is higher for cosmic ray with rigidity $\mathrm{R} \leq 100 \mathrm{GV}$ ( $\mathrm{GV}$ is a unit of rigidity, $\mathrm{GeV}$ is a unit of energy, for protons $1 \mathrm{GV}=1$ $\mathrm{GeV}$ ). Polar regions are the places with lower geomagnetic rigidity cutoff.

Neutrons reaching the Earth's surface are principally produced in the Earth's atmosphere by the interaction of the GCRs with air nuclei at high altitudes and in the subsequent development of a hadronic cascade down through the atmosphere. The neutron flux depending on energy, altitude, and geomagnetic latitude [9], [20], [21]. The energy threshold de neutron production in protons-air interaction is $\sim 1000$ $\mathrm{MeV}$. Detectors of secondary cosmic-ray particles at ground level, such as the neutron monitors, closer to the magnetic poles allow detecting particles with significantly lower rigidity (energy).

Fig. 1 (top panel) shown the time profiles of the sunspotnumber, and at the bottom panel, is shown the monthly cosmicray variation, in the period from 1958 to 2019. The cosmic rays data is from the Moscow neutron monitor, located at an altitude of 200 meters and a latitude of $66.47^{\circ} \mathrm{N}$. A inverse relationship between them, is evident, as well as, at solar minima 2009 and 2020, the variation of the cosmic ray (intensity) is up to $10 \%$ higher than average value.

Besides, cosmogenic nuclide, such as $10 \mathrm{Be}$ and ${ }_{14} \mathrm{C}$ with half-lives $1.39 \times 10^{6}$ and $5.37 \times 10^{3}$ years, respectively, are produced in the Earth's atmosphere by the interaction of GCRs with the nuclei of nitrogen and oxygen. $10 \mathrm{Be}$ is transported to the Earth's surface through rainwater and accumulates on the surface of the ground on sediments and ice at polar regions, and decays by beta decay to stable boron isotope $(10 \mathrm{~B})$. While ${ }_{14} \mathrm{C}$ is produced in the atmosphere when thermal neutrons from cosmic-ray air showers are captured by ${ }_{14} \mathrm{~N}$ nuclei through the reaction $\mathrm{n}+{ }_{14} \mathrm{~N} \rightarrow{ }_{14} \mathrm{C}+\mathrm{p}$. All living organisms absorb the ${ }_{14} \mathrm{C}$, and it stopped when they died. Cosmogenic nuclides are a long established tool for determining the ages of biological and inorganic samples. The method is extensively used in archaeology and geology [22]. 
Therefore, the concentration of radiative isotopes, such as of $10 \mathrm{Be}$, in sediments and ice and ${ }_{14} \mathrm{C}$ in organic remains are indicators of the long-term variation of the GCRs intensity, i.e., they are extensively used as a proxy for the history of cosmic rays that reached Earth. The higher the intensity of the cosmic rays, higher the concentration of cosmogenic isotopes at Earth [23]-[29]. The measurements of ${ }_{14} \mathrm{C}$ and ${ }_{10 \mathrm{Be}}$ are sensitive to the cosmic-ray flux in the energy range most affected by solar modulation. Thus, the temporal profiles of ${ }_{10 \mathrm{Be}}$ and ${ }_{14} \mathrm{C}$ concentrations, vary inversely with solar activity.

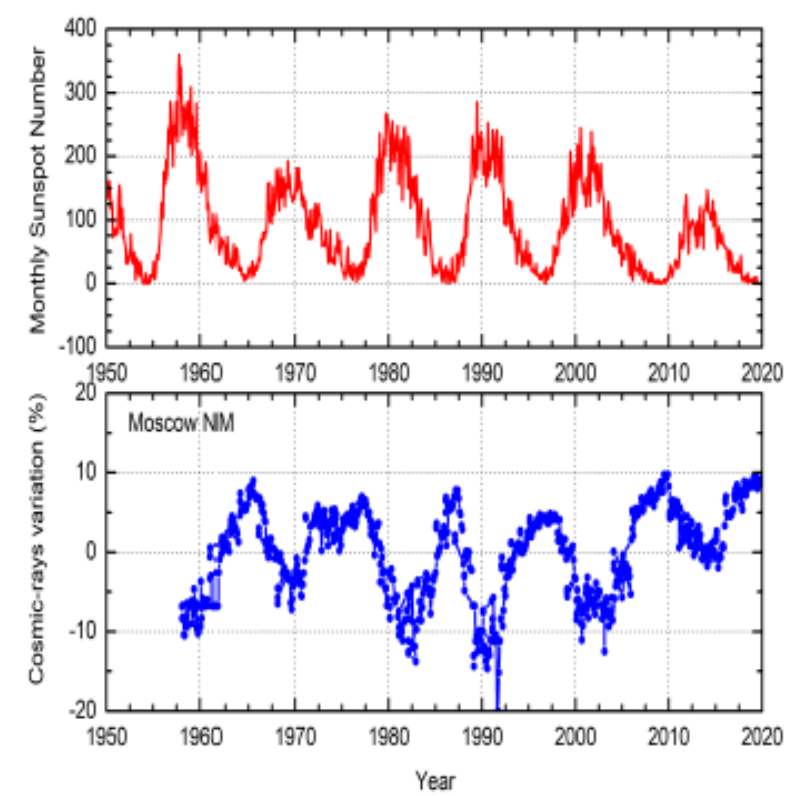

Fig. 1. Time profiles from 1958 to 2019: top panel, of the sunspot number, and bottom panel, of the secondary cosmic ray variation, observed at the Moscow neutron monitor, located at an altitude of 200 meters and a latitude of $66.47^{\circ} \mathrm{N}$.
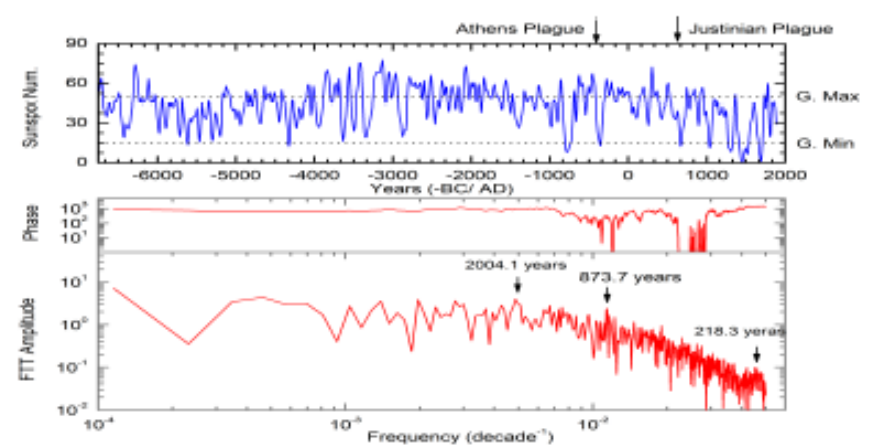

Fig. 2. The top panel shows the multi-isotope composite of sunspot number from -6755 (-BP) to 1885 (AD). The values are according to the WSN/ISN(v.1) definition [30]. The two vertical arrows indicate the occurrence of the Greek Prague and the Justinian Prague, respectively. They coincide with the epoch of two grand minima the Greek, and Roman. The bottom panel shows the phase and the amplitude as a function of the frequency of a fast Fourier transformation (FFT) analysis on the multiisotope composite data. The vertical arrows indicate the three most confidence periodicities from the study.

Fig. 2 (top panel) shows the temporal profile of the sunspot multi-isotope compound, of almost nine millennia data [30], since $-6755(-\mathrm{BP})$ to $1885(\mathrm{AD})$. The epochs where the sunspot number is no higher than 15 (marked by the horizontal line) are known as grand solar minima. Notice that in the last millennium, five grand minima of solar activity took place.

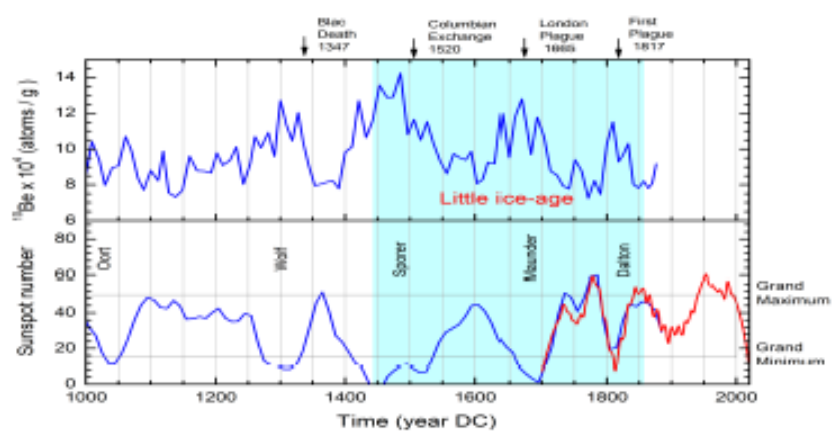

Fig. 3. The top panel shows the time profiles of the $10 \mathrm{Be}$ concentration, and the bottom panel represents the multi-isotope composite of sunspot number, both for the last millennium. The vertical labels represent the five last grand solar minima of the Holocene era. The vertical arrows indicate the occurrence of four historical pandemics.

The first records of the bubonic plague in the Christian era, known as the Justinian plague, were around $541 \mathrm{AC}$. However, after a century, its possible recurrence would kill $26 \%$ of the world population at the time. This period coincides with the first grand minimum of solar activity of the Christian era, known as the Roman Minimum. The vertical arrow (at right) in Fig. 2 (top panel) indicates its occurrence. Besides, the great Athens plague also coincides with the last grand minimum before the Christian era, known as the Athens Minimum, indicated by the vertical arrow (at left) in Fig. 2 (top panel).

The bottom panel shows a fast Fourier transformation (FFT) analysis, on the yearly decades' rates, from these almost nine millennia of sunspot numbers from multi-isotope data. The output represents the phase and amplitude as a function of frequency. The analysis shows only the signal of three solar cycles, of which the one with higher confidence, has a period of 873.7 years, representing the 1000-year Eddy cycle [18]. The second one has a period of 218.3 years and represents the 207-year Suess cycle [17]. The third has low confidence and appears with a period of 2004.1 years. This last period is not very far from the so-called Bray solar cycle [31], proposed as a climate cycle that correlated glacial advances around the world on the Holocene era, with a period of $2400 \pm 200$ years.

On the other hand, the high-resolution of the measurements of the $10 \mathrm{Be}$ concentration on the Dome Fuji ice core (Antarctica) [32], allows obtaining precise information on the GCRs intensity, reaching Earth over the past 1200 years, from 700 to 1900 , AC. Fig. 3 (top panel) shows the temporal profile of the concentration of $10 \mathrm{Be}$ on the Dome Fuji ice core during the last millennium. High $10 \mathrm{Be}$ concentration (peaks) correspond to grand solar minima, as indicate in Fig. 3 (bottom panel) where the time profiles of the sunspot multiisotope component [30], during the last 1000 years is shown. The five last grand solar minima from the Holocene era are in evidence [18], [24]; the last is the Dalton minimum also observed in the Wolfsunspot data. The Little ice-age period appears prominently in the time profiles. The red curve on the right side represents the smoothing number of sunspots observed from 1700 to 2000 . They overlap to the reconstruction of the sunspot number from cosmogenic isotopes, showing that the restoration has high confidence. 
In these periods, there was the occurrence of four historical pandemics. From left to right, the vertical arrows represent the bubonic Black Death (1347), within the Wolf minimum, the Smallpox or Columbian Exchange (1520), within the Sp• orer minimum; the bubonic plague of London (1665), within the Maunder minimum, and the First cholera plague (1817) within the Dalton minimum, respectively. These pandemics are reported extensively in the literature https://www.history.com/topics/middle-ages/pandemicstimeline.

There is a consensus that the Smallpox or Columbian Exchange plague (with onset on 1520) was passed along to the native American populations (without previous contact with diseases) by the Europeans. The death of millions of natives would have affected the climate, and the Earth would have cooled by the fall of agriculture [33]. However, the coincidence with the $\mathrm{Sp} \bullet$ orer minimum could explain better the Earth's cooling in that period and potentiating the plague decimation. The 1520 year is close to the onset of the socalled "little-ice age" (see also Fig. 3)

\section{B. In situ solar activity observations and pandemics}

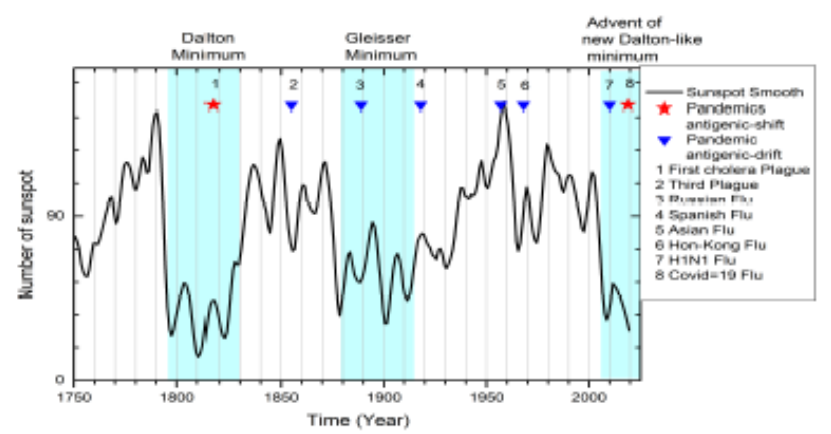

Fig. 4. Temporal profiles of sunspot from 1750 to 2019, the black curve is a smoothing of the sunspot data. The red stars and blue triangles indicate the date (year) of occurrence of pandemics due to antigenic-shift and antigenicdrift, respectively. The light-blue areas indicate the grand Dalton minimum, and the extensive but less intense Gleissberg solar minimum, respectively. The last triangle and star (H1N1 and Covid-19) are probably within a new grand solar minimum (see section II-C).

From 1749 the sunspot number is extensively recording and can be used for scientific purposes. The beginning of these observations coincides with the last years of the grand Mauder solar minimum and include the full grand Dalton solar Minimum. There is an overlap between sunspot data and reconstructed sunspots from cosmogenic isotopes, as shown in Fig. 3 (bottom panel).

One way to look for long periods of lesser solar activity from the data available of the sunspots is to create the smooth on the time-profiles. In statistics data processing, to smooth a data set is to create an approximating function that attempts to capture important patterns in the data, reducing the noise. Data smoothing can be used to help predict trends, and it is a tool available on most data analysis software. The smoothed sunspot data is shown in Fig. 4; the figure includes dates (red stars numbered as 1 and 8) from well-established outbreaks and classified as real pandemics due to their extent.

The analysis shows that there are three well defined long periods of lower solar activity, The first pandemic, red star 1, is within the grand Dalton minimum, and the red star 8 a pandemic due to a new virus type, the Covid-19, it is within the advent of a new Dalton-like minimum. Probably, only these two pandemics are linked with an antigenic-shift.

Sometimes, viruses from antigenic-drift may spill over to other species, and they can evolve to highly pathogenic plagues and pandemic in humans [34]. The blue triangles: 3 (Russian flu), 4 (Spanish flu), 5(Asian flu), 6(Hong-Kong flu), and 7 (Swine-flu), are in the category of antigenic drift, and the new strains of linfluenza virus, gain the ability to be hosted by other species, including humans.

Notice that the pandemic marked by the first star and labeled as First cholera plague also is in Fig. 3, it is the First plague 1817, within the grand Dalton solar minimum.

Besides, the Earth's magnetic field is nor constant over time. The global average magnetic strength over the last 200 years has decreased around $9 \%$, meaning that it is weakening. The confirmation of this behavior comes from recent observations from Swarm satellites from the European Space Agency (ESA) https: //www.esa.int/Applications/Observing the Earth/Swarm/ Swarm probes weakening of Earth $s$ magnetic field.

Therefore, it is expected that it is having an increase in the GCRs reaching the atmosphere, increasing the flux of neutrons. Perhaps this can explain the high rate of pandemics in the last 100 years.

\section{Some predictions}

The Space Weather Prediction Center from NOAA has released the latest forecast (December 2019) for sunspot Solar Cycle 25. Fig. 5 shows the forecast to Cycle 25 will be average in magnetic intensity and similar to Cycle 24. Cycle 25 will last up to about 2032. It will be a period of low magnetic activity, which is very similar to the observed at the end of Cycle 24.

The NOAA prediction for Solar Cycle 25, strongly confirms the advent of a Dalton-like minimum. This new Dalton-like period could be from 2006 to 2035, as shown in Fig. 6. The red curve represents the sunspot progression for Solar Cycles 3, 4, 5, 6. While the black curve shows the sequence for the Solar Cycle 22, 23, 24, the NOAA forecast of Solar Cycle 25 is similar to Solar Cycle 6, with this, Dalton-like minimum is reproduced again. The two black squares in Fig. 6 represent the dates of the two pandemics, the H1N1 (2009) (due to an antigenic-drift) and the Covid-19 (2019) (due to an antigenicshift), that already occurred in the new Dalton-like Minimum.

This similarity suggests the possibility that other pandemics outbreaks will happen specially those link with antigenic drift that are more common, up to 2035. By the way, the next solar minimum on 2031-2032 will be almost similar to the solar minimum in 2020. 


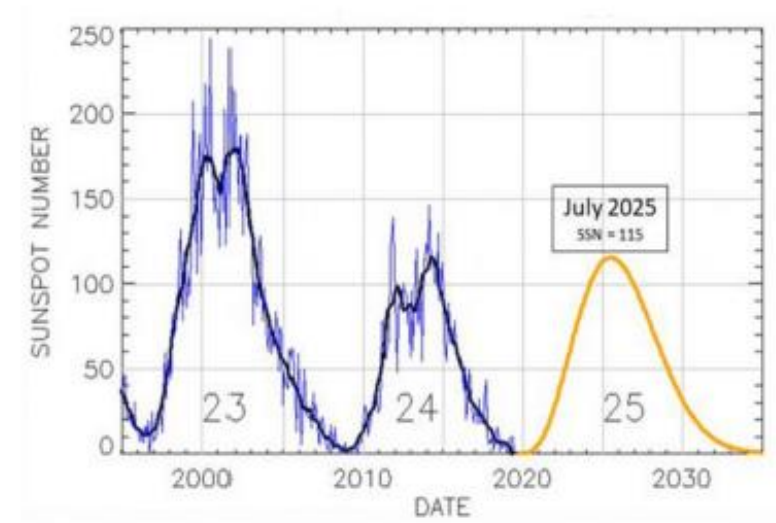

Fig. 5. Forecast (December 2019) for the sunspots Solar Cycle 25, according to the Space Weather Prediction Center from NOAA.

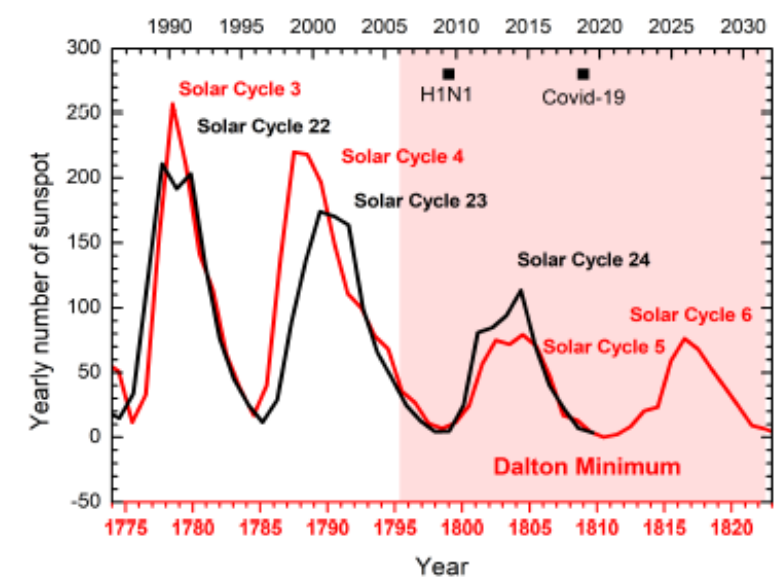

Fig. 6 . The figure shows the similarity in intensity and duration between solar cycles 3, 4, 5, and 6 (lower red scale) and Solar cycles 22, 23, and 24 (upper black scale). If the NOAA forecast to Cycl2 25 was included, we would have the advent a period such as the Dalton-like minimum, again Notice that the H1N1 and Covid-19 flu pandemics (black squares) would already be part of this new Dalton-like solar minimum.

\section{Mutations By Cosmic-Neutrons}

Mutations are induced on a biological system via several agents, such as temperature shocks or chemical products that change the $\mathrm{pH}$ value (acidity). However, mutations can also be induced by ionizing electromagnetic radiation, $\mathrm{X}$ rays, and $\gamma$-rays, as well as by corpuscular radiation, such as neutrons or charged particles as protons and alpha-rays. Although UV radiation does not produce ionization, but cause excitation through energy transfer and can induce mutations. At Earth, the UV radiation is restricted to the higher atmosphere. The ozone layer absorbs it.

In the '30s, cosmic-ray induced mutations as the origin of a grant variety of species have been suggested by [35]. It has also been suggested that natural radioactivity is unable to induce mutations and that cosmic radiation could be one of the factors determining mutations [36]. In general, the cellular response to cosmic rays, including mutations, are detailed by [37], [38] and references therein.

The global population-weighted annual effective dose due to cosmic-ray exposure is $0.34 \mathrm{mSv}$ [39], $19.6 \%$ correspond to neutrons, $4.6 \%$ to protons, $8.4 \%$ to electrons, $7.9 \%$ to photons and $59 \%$ to muons. We can see that more than $50 \%$ correspond to muons. Indeed, the muons are most abundant at ground level, especially at sea level. Still, they are less likely to induce mutations than other ionizing radiation because they deposit less energy per unit of track length. In other words, their absorption length is much higher than other ionizing radiation, such as protons, electrons, and so on. Also, the amount of fast protons at ground level is comparable to the neutrons. However, both protons and the atomic nuclei at the proteins have a positive electrical charge; the repulsive force between them prevents them from being captured. In most cases, electrically charged particles are scattered elastically by atomic nuclei. Only neutrons from cosmic rays can be thermalized for collisions with the air molecules, increasing the capture crosssection by atomic nuclei, and their lateral expansion. They are the dominant particles, to produce mutations (antigenic-shift) at ground level.

The effect of neutrons depends upon the presence of hydrogen in the tissues [40]. If a neutron collides with a proton (hydrogen) from a viral cell, it tends to hit in more than one place. While the majority of the neutrons not be absorbed or deflected, and they pass right through the small size of cells without producing any effects on them. Yet, a fraction of them can be scattered through an interaction with the hydrogen of the proteins mainly on the virus's surface, and can produce small changes, called "antigenic-drift". The change is primarily on antigenically surface proteins, forming a diversity of reassortant virus subtype, and in most cases, they cause mild infections in their natural hosts, with seasonal variations.

The capture of a neutron within the cell can produce an ion trail in the path of the recoil protons, creating a radical change, a new subtype of the virus, this change is called "antigenic-shift", forming some reassortant strains of viruses and some times a new lineages of virus [41]. That new virus lineage can cause, in most cases, high pathogenic effects.

The occurrence rates of the two above described processes depend strongly on the neutron velocity (kinetic energy). Thermal neutrons are those whose average kinetic energy is equal to the kinetic energy of air molecules at environment temperatures, and this kinetic energy is about $0.03 \mathrm{eV}$ (at $20^{\circ}$ c). The neutron capture cross-section is higher by thermal neutron than fast neutrons. Besides, the flow at the ground level of thermal neutrons is about 6-7 times smaller than the flux of the fast neutrons (kinetic energies of about $10 \mathrm{MeV}$ ) [42].

The cross-section of thermal neutron capture $\left(\sigma_{c}\right)$ increases as the speed (kinetic energy) of the neutron decreases, following law $1 / \mathrm{v}$. However, the scattering cross-section $\left(\sigma_{\mathrm{s}}\right)$ of fast neutrons is about 100 times larger than the capture crosssection of thermal neutrons.

Our central assumption is to consider that the neutron capture cross-section is proportional to the probability of a neutron to induce an antigenic-shift. The neutron scattering cross-section is proportional to the probability of a neutron to produce an antigenic-drift.

$$
\frac{\text { antigenic-shift-probability }}{\text { antigenic-drift-probability }}=\frac{\sigma_{c}}{\sigma_{s}} \begin{cases}10^{-2} & \text { thermal neutrons } \\ 10^{-5} & \text { fast neutrons }\end{cases}
$$

The above ratios considerate the atomic hydrogen nucleus as the target, values for other targets such as carbon and oxygen are negligible. Because amino acids constitute 
antigenic proteins in viruses, and their composition is mainly carbon $(\mathrm{C})$, hydrogen $(\mathrm{H})$, oxygen $(\mathrm{O})$, and nitrogen $(\mathrm{N})$, However, the antigenic-drift and especially the antigenicshift linked with neutron capture cross-section is relevant only for hydrogen, and negligible for the other elements.

Thus, the probabilities ratio of antigenic-shift to antigenicdrift, for thermal and fast neutrons, can be obtained from their respective cross sections. They are well-known quantities, and available at JANIS software, http://www.oecd-nea.org/janis/. So the probability that the neutron will induced an antigenicshift (mutation) is

$$
\text { antigenic-shift-probability }=\frac{\sigma_{c}}{\sigma_{c}+\sigma_{S}},
$$

The values of this probability, for thermal and fast neutrons and the $\mathrm{H}, \mathrm{C}$, and $\mathrm{O}$ atomic nuclei as the target are summarized in Table I.

TABLE I: ANTIGENIC-SHIFT PROBABILITY, IN THE AMINO ACID ELEMENTS OF ANTIGENIC VIRAL PROTEINS

\begin{tabular}{ccc}
\hline \hline \multicolumn{3}{c}{ antigenic-shift-probability } \\
\hline Target & Thermal neutrons & Fast neutrons \\
\hline $\mathrm{H}$ & $8.3 \times 10^{-3}$ & $1.7 \times 10^{-6}$ \\
$\mathrm{C}$ & $2.9 \times 10^{-4}$ & $1.4 \times 10^{-6}$ \\
$\mathrm{O}$ & $1.4 \times 10^{-5}$ & $4.3 \times 10^{-9}$ \\
\hline \hline
\end{tabular}

Thermal neutrons exhibit essential properties, such as an increase in the cross-section for their capture by $14 \mathrm{~N}$, inducing the formation of various radioactive isotopes such as ${ }_{14} \mathrm{C}$. Still, also only thermal neutrons can the capacity of producing antigenic-shift in viruses because antigenic-shift by fast neutrons and other secondary cosmic ray particles, such as protons and electrons, is negligible. Practically just the hydrogen nuclei within the virus have effectiveness as targets for thermal neutrons to induce an antigenic-shift.

A variety of animals hosts the viruses; on average, the radiation dose by cosmic ray exposure is determined considering the human body as a target. Determining the effects of cosmic-rays on biological systems requires measuring its energy spectrum $\varphi(E)$. It depends on geomagnetic latitude and altitude of places where it is measured. An analytical expression to the cosmic-ray neutron dose rate is obtained, integrating the ratio between the neutron energy spectrum $\varphi(E)$ and the conversion factor $\mathrm{g}(\mathrm{E})$ [9].

However, more robust values for the cosmic-ray dose rate can be obtained from models incorporated in Monte Carlo calculations, such as the CARI-6 model [43]. A more sophisticated model, such as the EXPACS [39], takes into account variations with the solar cycle, altitude, and magnetic latitude.

Fig. 7 (top curves) shows the evaluation of European population dose rates due to cosmic-ray exposure with the altitude and for three different geomagnetic rigidity cutoff (magnetic latitude), obtained from the EXPACS model [43], including the period from 2005 to 2016 . This period includes a complete solar cycle. These values vary with the solar modulation condition within a range of approximately $15 \%$ [39].

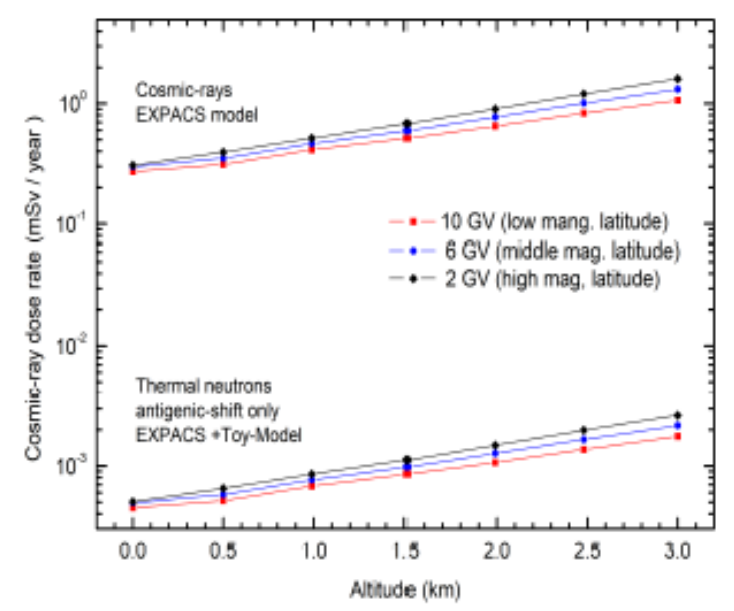

Fig. 7. Top curves: Evaluation of European population effective annual dose rate due to cosmic ray exposure, estimated using EXPACS model as a function of altitude and considering different vertical cut-off rigidity (geomagnetic latitude) [43]. Bottom curves: the same as the curve at the top, but considering only the thermal neutrons inducing antigenic-shifts, obtained in this work (Toy-model). These values vary with the solar modulation condition within a range of approximately $15 \%$.

Now, if we consider the probability of neutron capture inducing an antigenic change, according to the values of Table I. The radiation dose rate, by thermal neutrons inducing antigenic-shift, can be estimated from the global cosmic-ray dose rate. The bottom curves in Fig. 7 summarize the results.

Because the radiation dose rate per year, by thermal neutrons inducing an antigenic-shift, is around 1000 times less than an antigenic-drift inducing by cosmic rays.. Long periods of low solar activity are necessaries to a substantial increase of the thermal neutrons flow to produce an antigenicshift, triggering pandemics.

\section{Two-Dimensional ANALYSis Of EPIDEMICS}

Small changes associated with antigenic-drift can accumulate over time and result in viruses that are antigenically different. The body's immune system may not recognize these newer viruses producing new viral outbreaks. These epidemics have occurrence rates that are influenced by seasonal environmental conditions. However, we also show the 11-year solar cycle solar activity modulating these viral outbreaks. This behavior and the high occurrence rates suggest that the scattering of cosmic rays induces the antigenic-drifts. Indeed, the total cosmic-ray dose rate is about 1000 times higher than the neutron dose rate due to neutron capture (antigenic-shift).

If the long-term data of more common viral outbreaks have other pieces of information beyond their date of occurrence, always obtained with the same systematic to get reasonable confidence, it is possible to do a two-dimensional analysis. However, comparing two sets of data, point by point, to see if there is any correlation between them may be impractical due to the noise embedded in both. As already commented, one way to decrease noise from a data set is to use an existing algorithm in most data analysis software, known as smoothing. Data smoothing can be used to help predict trends.

Fig. 8 shows the number of people infected with measles in Glasgow over time, left scale, and the progression of the 
number of sunspots, from 1888 to 1927 , right scale [44]. Following Fig. 8, one can see that there is a trend, an oscillation in the number of measles cases out of phase with the sunspot number oscillation (an almost inverse relationship between them). In other words, the oscillation of the sunspot number train is inverse with the oscillation of cosmic-neutron flow, inducing an oscillation in the antigenic-drift in the measles virus. Notice that the analysis does not take into account other variations, such as seasonality from the environment. We have used old data because the incidence of measles underwent a radical change in 1963 with the advent of the vaccine. The number of measles cases dropped by $99 \%$, but there are still outbreaks.

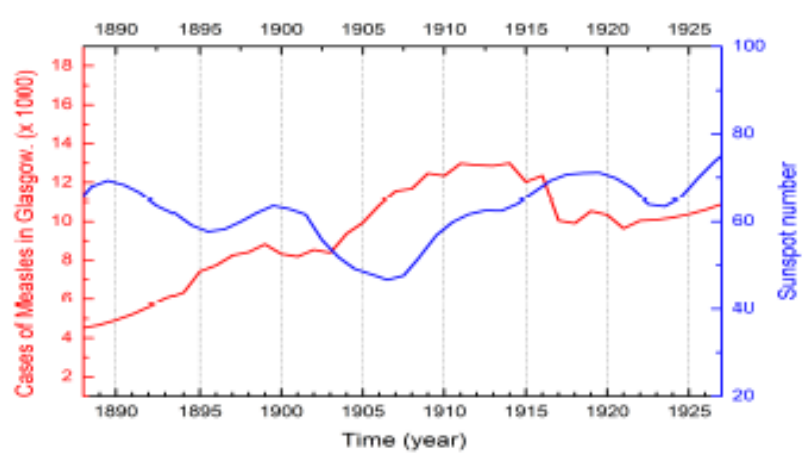

Fig. 8. The red curve (left scale) shows the oscillation train of the number of people infected with measles in Glasgow, from 1888 to 1927, [44], The blue curve (right scale) shows the oscillation train of the number of sunspots, for the same period. We can see an almost inverse relationship between them.

Also, long-term data of deaths from influenza and pneumonia in the United States of America, starting in 1950, are available

https://www.statista.com/statistics/184574/deaths-byinfluenza-and-pneumonia-in-the-us-since-1950/, but the annual rates, are only available from 1995 onwards. The data is consistent with an exponential fall of deaths over time, due to prevention and surveillance programs through vaccination campaigns. The trend is a kind of damped oscillation in an inverse relationship with the sunspot train. Fig. 9 shows the results. The damped oscillation of the deaths for influenza in an inverse relationship with the sunspots oscillation, it is more a piece of evidence that the flu outbreaks can be associated with antigenic-drifts; triggered for the scattering of secondary cosmic-ray by the hydrogen nuclei in the viral proteins.

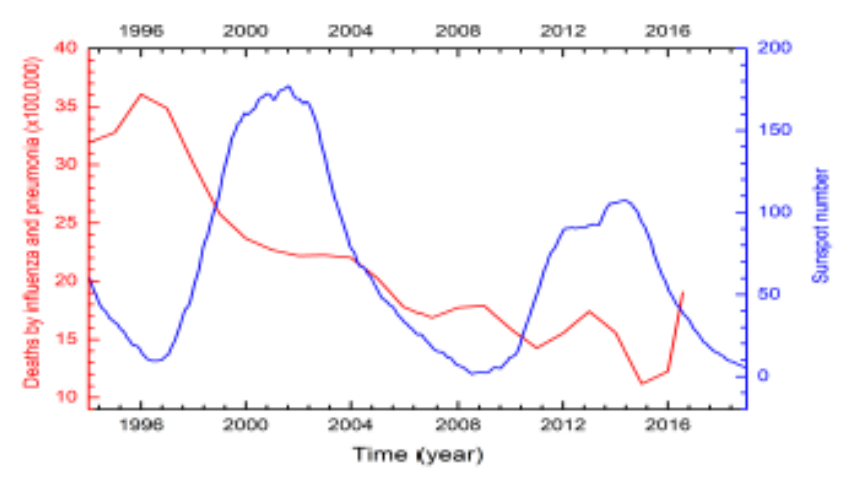

Fig. 9. The red curve (left scale) shows the damped oscillation of the annual number of deaths, from influenza and pneumonia in the United States of America, starting in 1995. The damped is due to a gradual increase in the prevention and surveillance programs through vaccination. The blue curve (right scale) shows the oscillation train of the number of sunspots, for the same period. We can see an almost inverse relationship between them.

\section{Conclusions}

We show that the GCRs are the dominant source of neutron flow at Earth. The cosmogenic-isotopes concentration, such as the ${ }_{14} \mathrm{C}$, and $10 \mathrm{Be}$, are indicators of the long-term variation of the GCRs flow at Earth [23], [24], [27]-[29]. From these studies, we can see that the Earth would have gone through several cooling periods, i.e., periods of reduced solar activity and long periods when the number of sunspots does not exceed 15. These periods are known as the grand solar minima. As the GCRs are in an inverse relationship with solar activity, during the grand solar minima, GCRs flow reaching Earth is highest.

We show that the atomic hydrogen nuclei in the viruses are the most effective targets for viral mutations induced by cosmic-neutrons. We link the thermal neutron capture crosssection with viral antigenic-shift. The low rate of antigenicshift causing radical changes in the viruses suggests that they can trigger pandemics. Since humanity still has no immunity to these new viral strains, which facilitates its propagation and dissemination. As the thermal-neutron radiation dose rate inducing antigenic-shift is around 1000 times smaller than the cosmic-ray dose rates causing antigenic-drift (see Fig. 7). The viral antigenic-shift occurrence requires a substantial increase in the thermal neutron flows at Earth. It is more feasible during the grand solar minima when the GCRs flow, and consequently, the thermal neutrons flow is the highest.

We show that the historical pandemics of humanity coincided with the last seven grand solar minima. It is probably that the high rate of viral pandemics in the last 200 years be a consequence of the weakening of the Earth's magnetic field as confirmed by the ESA Swarm satellites, increasing the GCRs hitting the Earth and consequently the thermal neutron flow at ground level.

Also, the scattering cross-section from cosmic rays, including the fast and thermal neutrons, would be linked with viral antigenic-drift. The high rate of antigenic-drift causing small changes on the viruses suggests that they can trigger the emergence of viral epidemics but, in most cases producing infections in their mildest form. They are also subject to seasonal variations. We also show that the 11-year solar cycle activity, modulate viral outbreaks linked with antigenic-drift.

The latest forecast (December 2019) of NOAA for the Solar Cycle 25., showing that it will be of small intensity and similar to Cycle 24., strongly suggest the advent of a new Dalton-like minimum, with onset on 2006 and probably lasting up to 2035. This new minimum already has the register of two pandemics, H1N1 (2009) and Covil-19 (2019) and the probability of emergence of a new is not small, especially in the next 11-year solar minimum 2030-2033.

\section{ACKNOWLEDGMENT}

We express our gratitude to all open access data catalogs used in this work. This work is supported by the Rio de Janeiro Research Foundation (FAPERJ), under Grants 08458.009577/2011-81.

\section{REFERENCES}

[1] R. Hope-Simpson, "Sunspots and flu: a correlation", Nature, vol. 275, pp. 86, September 1978.

[2] F. Hoyle, and N. Wickramassinghe, "Sunspots and influenza", Nature, vol. 343, pp. 304, January 1990 
[3] S. Ertel, "Influenza pandemics and sunspots : easing the controversy", Naturwissenschafte, vol. 81, pp. 308-311, 1994.

[4] S. Tower, "Sunspot activity and influenza pandemics: a statistical assessment of the purported association", Epidemiology \& Infection, vol. 145 , pp. 2640-2655, October 2017.

[5] D. J. D. Earn, J. Dushoff, and S. A. Levin, "Ecology and Evolution of the Flu", Trends in Ecology and Evolution, vol. 17, pp. 334-340, July 2002 .

[6] S. Caini et al. "Climatic factors and long-term trends of influenza-like illness rates in The Netherlands, 1970-2016", Environmental Research, vol. 167, pp. 307-313, July 2018.

[7] J. Treanor, "Influenza vaccine-outmaneuvering antigenic shift and drift", New England Journal of Medicine, vol. 350, pp. 218-220, January 2004.

[8] M. Nagal, and G. L. Locher, "Production of Mutations by Neutrons", Nature,vol. 140, pp. 111-112, July 1937.

[9] T. Nakamura, Y. Uwamino, T. Ohkubo, and A. Hara, "Altitude variation of cosmic-ray neutrons", Health Physics, vol. 53, pp. 509517, October 1987.

[10] J. F. Ziegler et al., "IBM experiments in soft fails in computer electronics (1978-1994)", IBM Journal of Research and Development, vol. 40, pp. 3, January 1996

[11] J. Granger, L. Walkowicz, R. Fitak, and S. Johnsen, "Gray whales strand more often on days with increased levels of atmospheric radiofrequency noise", Current Biology, vol. 30, pp. 155-156, February 2020.

[12] N. G. Ptitsyna et al., "Possible effect of geomagnetic disturbances on the incidence of traffic accidents (St. Petersburg 1987-1989)", Phys Med., vol. 11, pp. 93-101, 1995.

[13] N. G. Ptitsyna et al., "Natural and man-made low-frequency magnetic fields as a potential health hazard", Physics-Uspekhi, vol. 41, pp. 687 709, July 1998

[14] S. M. Tobias, "The Solar Dynamo", Philosophical Transactions of the Royal Society A, vol. 360, pp. 2741-2756, October 2002.

[15] T. Turner, G. Swindles, G., D. Charman et al., "Solar cycles or random processes? Evaluating solar variability in Holocene climate records", Sci Rep., vol. 6, 23961, June 2016

[16] A. N. Peristykh, and P. E. Damon, "Persistence of the Gleissberg 88year solar cycle over the last 12,000 years: Evidence from cosmogenic isotopes", J. Geophys. Res., vol. 108, pp.1-15, July 2002.

[17] H. E. Suess, "The radiocarbon record in tree rings of the last 8000 years", Radiocarbon, vol. 22, p. 200-209, July 1980

[18] J. A. Eddy, "The Maunder Minimum", Science, vol. 192, pp-11891202, June 1976.

[19] M. N. de Oliveira, C. R. A. Augusto, C. E. Navia, and A. A. Nepomuceno, "On the short term modulation of cosmic rays by highspeed streams at the Pierre Auger surface array detectors", arXiv:2003.06432v1 [astro-ph.HE], 2020.

[20] T. Nakamura, T. "Cosmic-ray Neutron Spectrometry and Dosimetry", Journal of Nuclear Science and Technology, vol. 45, pp. 1-8, June 2008.

[21] A. C. Overholt, A. L. Melott, and D. Atri, "Modeling cosmic ray proton induced terrestrial neutron flux: A look-up table", J. Geophys. Res. Space Physics, vol. 118, pp. 2765-2770, June 2013.

[22] E. C. Anderson, W. F. Libby, S. Weinhouse, A. F. Reid, A. D.Kirshenbaum, and A. V. Grosse, "Radiocarbon from cosmic radiation”, Science. vol. 105, pp. 576-577, May 1947.

[23] J. A. Eddy, "The case of the missing sunspots", Scientific American, vol. 236, pp. 80-95, May 1977https://www.jstor.org/stable/24954027.

[24] M. Stuiver, T. F. Braziunas, T. F. 1988, The solar component of the atmospheric $14 \mathrm{C}$ record, in: F. R. Stephenson, A. W. Wolfendale (Eds.), Secular Solar and Geomagnetic Variations in the Last 10,000 Years. Kluwer publs, Dortecht, The Netherlands, p. 245, 1988.

[25] D. V. Hoyt, and K. H. Schatten, "Group Sunspot Numbers: A New Solar Activity Reconstruction”, Solar Physics, vol. 179, pp. 189-219, April 1998.

[26] K. G. McCracken, F. B. McDonald, J. Beer, G. Raisbeck, and F. Yiou, "A phenomenological study of the long-term cosmic ray modulation, 850-1958 AD”, J. Geophys. Res., vol. 109, A12103, December 2004

[27] I. G. Usoskin, S. K. Solanki, and G. A. Kovaltsov, "Grand minima and maxima of solar activity: new observational constraints", A\&A, vol. 471, pp. 301-309, August 2007.

[28] I. G. Usoskin et al., "Evidence for distinct modes of solar activity", A\&A, vol. 562, L10, 2014.

[29] F. Clette, L. Svalgaard, J. M. Vaquero, and E. W. Cliver, The Solar Activity Cycle ed A. Balogh et al, New York: Springer 35, 2015.

[30] C. J. Wu, I. G. Usoskin, N. Krivova, G. A. Kovaltsov, M. Baroni, E Bard, and S. K. Solanki1, "Solar activity over nine millennia: A consistent multi-proxy reconstruction", A\&A, vol. 615, A93, July 2018 .
[31] S. S. Vasiliev, and V. A. Dergachev, "The 2400-year cycle in atmospheric radiocarbon concentration: bispectrum of $14 \mathrm{C}$ data over the last 8000 years", Annales Geophysicae, European Geosciences Union, vol. 20, pp. 115-120, 2002.

[32] K. Horiuchi, T. Uchida, Y. Sakamoto, A. Ohta, H. Matsuzaki, Y. Shibata, and H. Motoyama, "Ice core record of $10 \mathrm{Be}$ over the past millennium from Dome Fuji, Antarctica: A new proxy record of past solar activity and a powerful tool for stratigraphic dating", Quaternary Geochronology, vol. 3, pp. 253-261, August 2008.

[33] A. Koch, C. Brierley, M. M. Maslin, and S. A. Lewis, "Earth systemimpacts of the European arrival and Great Dying in the Americas after 1492", Quaternary Science Reviews, vol. 207, pp. 13 36, March 2019.

[34] R. G. Webster, W. J. Bean, O. T. Gorman, T. M. Chambers, and Y. Kawaoka, "Evolution and ecology of influenza A viruses", Microbiol. Rev., vol. 56, pp. 152-179, 1992.

[35] M. Delbr"uck, and N. W. Timof'eeff-Ressovsky, "Cosmic rays and the origin of species", Nature, vol. 137, pp. 358-359, February 1936.

[36] H. J. Muller, and L. M. Mott-Smith, "Evidence that natura radiactivity is inadequate to explain the frequency of natural mutations", Proc. nat. Acad. Sci., U.S.A., vol. 16, pp. 277-285, April 1930.

[37] G. Duell, and B. Duell, B. "Organic Responses to Cosmic Rays and Their Secondaries", Recent Studies in Bioclimatology, pp. 61-67, 1954

[38] D. Atri, and A. L. Melott, "Biological implications of high-energy cosmic ray induced muon flux in the extragalactic shock model",Geophysical Research Lett., vol. 38, L19203, October 2011.

[39] T. Sato, "Evaluation of World Population- Weighted Effective Dose due to Cosmic Ray Exposure", Scientific Reports, vol. 6, 33932, September 2016.

[40] M. A. Nagai, and G. L. Locher, "The Production of Mutations in Drosophila with Neutron Radiation", Genetics, vol 23, pp. 179-189, March 1938.

[41] M. Liu et al., "Antigenic Patterns and Evolution of the Human Influenza A (H1N1) Virus", Sci Rep., vol. 5, 14171, 2015.

[42] M. Yamashita, L. D. Stephens, and H. W. Patterson, "Cosmic-RayProduced Neutrons at Ground Level: Neutron Production Rate and Flux Distribution”, J. Geophys. Research, vol. 71, pp. 3817-3834, August 1966

[43] G. Cinelli et al., "European annual cosmic-ray dose: estimation of population exposure", J. Maps, vol. 13, pp. 812-821, 2017.

[44] H. E. Soper, H. E. 1929, "The Interpretation of Periodicity in Disease Prevalence", Journal of the Royal Statistical Society, vol. 92, pp. 34 73,1929 